\title{
PADRONIZAÇÃO DA SÍNTESE DOS PRINCIPAIS CONSTITUINTES FEROMONAIS DE INSETOS DOS GÊNEROS METAMASIUS E RHYNCHOPHORUS
}

\author{
J. D. Freitas ${ }^{1}$, S. K. M. Cavalcante ${ }^{1}$, E. D. SANTOS ${ }^{1}$, L. O. Antunes ${ }^{1}$, A. J. D. Freitas ${ }^{1}$, \\ M. L. Freitas ${ }^{2}$, H. F. Goulard ${ }^{2}$, A. E. G. Santana ${ }^{2}$ \\ ${ }^{1}$ Curso de Química do Instituto Federal de Alagoas (IFAL) \\ johnnatan.duarte@ifal.edu.br \\ ${ }^{2}$ Instituto de Química e Biotecnologia da Universidade Federal de Alagoas (UFAL)
}

Artigo submetido em janeiro/2011 e aceito em abril/2011

\section{RESUMO}

Os curculionídeos são insetos que causam grandes problemas econômicos aos agricultores e atingem culturas, como coco, dendê, cana-de-açúcar, milho, banana, algodão, etc. O controle de pragas agrícolas, ainda hoje, utiliza grandes quantidades de pesticidas que são prejudiciais à saúde dos animais, do homem e do meio ambiente. Uma alternativa ao uso desses pesticidas é o uso de feromônios, por serem mais seletivos e menos tóxicos. Nesse trabalho, descrevemos a síntese, em escala preparativa, dos compostos ( \pm )-5-Nonanol, $( \pm)$-2-Metil-4octanol, ( \pm )-2-Metil-4-heptanol, ( \pm )-3-Metil-4-octanol, ( \pm )-5-Metil-4-octanol, $( \pm)-4-M e t i l-5-$

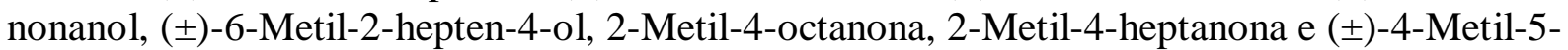
nonanona, principais constituintes feromonais de alguns insetos dos gêneros Metamasius e Rhynchophoruseas. A síntese consistiu na reação de Grignard entre um aldeído e um brometo de alquilmagnésio. As oxidações dos álcoois para obtenção das cetonas foram realizadas com hipoclorito de sódio.

PALAVRAS-CHAVE: Síntese orgânica, feromônios, curculionídeos.

\section{STANDARDIZATION SINTETIC OF THE MAJOR CONSTITUENTS OF THE INSECT PHEROMONES OF GENRES METAMASIUS AND RHYNCHOPHORUS}

\begin{abstract}
The weevils are insects that cause major economic problems for farmers and ancient cultures, such as coconut, palm oil, sugar cane, corn, bananas and cotton. The control of agricultural pests, even today, uses large amounts of pesticides that are harmful to the health of animals, humans and the environment. An alternative to the use of these pesticides is the use of pheromones, more selective and less toxic. In this paper we describe the synthesis, preparative scale, the compounds $( \pm)$-5-Nonanol, $( \pm)$-2-Methyl-4-octanol, $( \pm)$-2-Methyl-4-heptanol, $( \pm)$-3Methyl-4-octanol, ( \pm )-5-Methyl-4-octanol, ( \pm )-4-Methyl-5-nonanol, ( \pm )-6-Methyl-2-hepten4-ol, 2-Methyl-4-octanon, 2-Methyl-4-heptanone and ( \pm )-4-Methyl-5-nonanone, major constituents of the insect pheromones of genres Metamasius and Rhynchophorus. The synthesis consisted of the Grignard reaction between an aldehyde and a bromide alkyl magnesium. The oxidation of alcohols to obtain the ketones were performed with sodium hypochlorite.
\end{abstract}

KEY-WORDS: Organic synthesis, pheromones, weevils. 


\section{PADRONIZAÇÃO DA SÍNTESE DOS PRINCIPAIS CONSTITUINTES FEROMONAIS DE INSETOS DOS GÊNEROS METAMASIUS E RHYNCHOPHORUS}

\section{INTRODUÇÃO}

\section{OS INSETOS}

Antes do descobrimento do Brasil, nossos insetos se limitavam a destruir apenas plantas silvestres. Com a vinda dos colonizadores, diversas plantas foram introduzidas e parte dos insetos nativos passou a retirar o alimento dessas novas plantas. Tais plantas se apresentavam geralmente concentradas em uma determinada região. Assim sendo, alguns insetos encontraram ambiente adequado para sua reprodução, multiplicando-se rapidamente originando desta forma grandes populações, comprometendo então a produção agrícola em diversas partes do país. Além disso, houve a introdução acidental de muitos insetos-praga de regiões estrangeiras. Desta forma os problemas relacionados aos insetos fitófagos aumentam e se tornam mais complicados a cada dia (MARICONI, 1988).

Uma parte dos insetos introduzidos no Brasil jamais constituiu problema para nossas plantações, é o caso do piolho de São José, Quadraspidiotus perniciosus (Comstock, 1881) (Hemiptera: Diaspididae), praga da macieira (Pyrus malus L.), da pereira (Pyrus communis L.) e da ameixeira (Prunus domestica L.) (DAY, 2000), mas tem promovido grandes danos em várias outras regiões, como por exemplo na Califórnia nos Estados Unidos e parte da Europa (MARICONI, 1988).

Outra parte dos insetos introduzidos no Brasil tem tanta ou maior importância para nós, como praga, do que para o seu próprio país de origem. Dentre esses insetos podemos destacar a lagarta rosada do algodoeiro, Pectinophora gossypiella (Saunders, 1843) (Lepidoptera: Gelechiidae), que é um problema para todos os países produtores de algodão (Gossypium ssp.) e a broca do café, Hypothenemus hampei (Ferrari, 1867) (Coleoptera: Scolytidae) (DAMON, 2000; TEIXEIRA et al., 2006), que ocasiona maiores danos aqui no Brasil do que na sua região de origem, a África (MARICONI, 1988).

Devido aos grandes problemas econômicos ocasionados pelos insetos-praga, o homem, ainda hoje, utiliza os inseticidas como o principal grupo de compostos químicos responsáveis pelo controle destes na agricultura.

\section{A UTILIZAÇÃO DE PESTICIDAS}

Os insetos são considerados nossos maiores competidores no que diz respeito à alimentação. Nessa disputa o homem tem recorrido principalmente ao uso de pesticidas sintéticos tradicionais, como por exemplo, os compostos organoclorados, organofosforados e carbamatos. Tais pesticidas são bastante perigosos aos animais, ao homem e ao meio ambiente de modo geral.

Os pesticidas organoclorados têm amplo espectro de ação e se degradam lentamente, persistindo de meses a anos nos organismos. A toxicologia desses contaminantes é altamente complexa e específica para cada composto. Assim, pode haver múltiplas respostas tóxicas dependendo então da espécie, sexo e órgãos atingidos (VEIGA et al., 2006). Alguns exemplos desses pesticidas são: o Diclorodifeniltricloroetano, conhecido como DDT (1), o Clordano (2), o Aldrin (3) e o Dieldrin (4), mostrados na figura 1. 

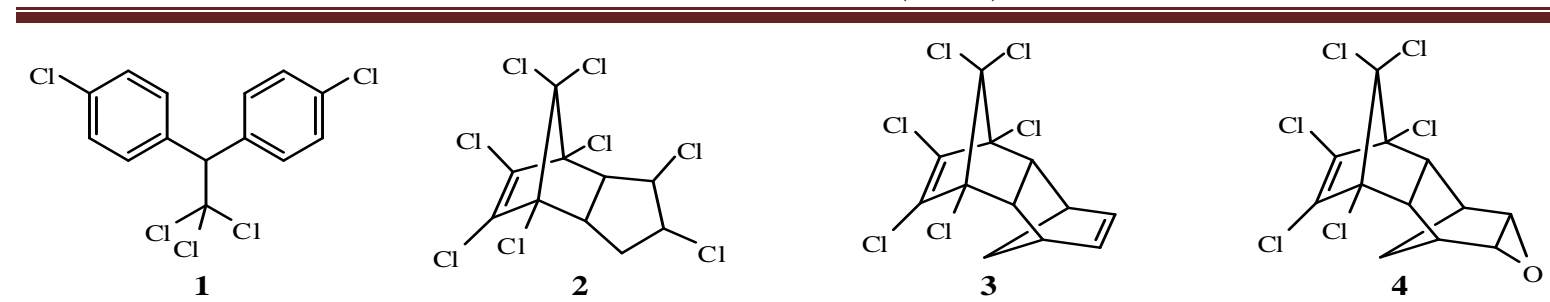

Figura 1 - Exemplos de pesticidas da classe dos organoclorados.

Os carbamatos são substâncias derivadas do Ácido Carbâmico (5) e apresentam alta ação inseticida. Os compostos desse grupo apresentam baixa ação residual, devido à instabilidade química das moléculas e baixa toxicidade por um longo período, quando comparada com os derivados fosforados.

A figura 2 mostra as estruturas do Ácido Carbâmico (5) e dos pesticidas Baygon ${ }^{\circledR}$ (6), Carbaril $^{\circledR}(\mathbf{7})$ e Carbofuran ${ }^{\circledR}(\mathbf{8})$ da classe dos carbamatos.<smiles>NC(=O)O</smiles><smiles>CNC(=O)Oc1ccccc1OC(C)C</smiles>

6<smiles>CNC(=O)Oc1cccc2ccccc12</smiles>

7<smiles>CNC(=O)Oc1cccc2c1OC(C)(C)C2</smiles>

8

Figura 2 - Estrutura do Ácido Carbâmico e de alguns pesticidas da classe dos carbamatos.

Os pesticidas organofosforados são compostos orgânicos derivados do Ácido Fosfórico (9) e foram desenvolvidos durante a Segunda Guerra Mundial. Tais compostos são muito perigosos, pois são tóxicos não apenas para insetos, mas também para animais. Sua toxicidade para humanos pode ser comparada à estricnina, arsênio e cianetos. Porém, não persistem tanto tempo no meio ambiente quanto os organoclorados por serem hidrolisados mais facilmente (WARE, 1989; SANTOS et al., 2007).

A figura 3 mostra as estruturas do Ácido Fosfórico (9) e dos pesticidas Diclorvos (10), Paration (11) e Malation (12) da classe dos organofosforados:

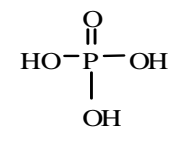<smiles>COP(=O)(OC)OC=C(Cl)Cl</smiles>

10

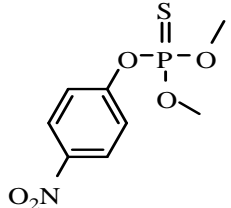

11<smiles>CCOC(=O)CC(SP(=S)(OC)OC)C(=O)OCC</smiles>

12

Figura 3 - Estrutura do Ácido fosfórico e de alguns pesticidas da classe dos organofosforados.

\section{OS PROBLEMAS OCASIONADOS PELO MAU USO DE PESTICIDAS}

Devido ao longo espectro da ação dos pesticidas, associado ao seu uso indiscriminado, surgiram várias complicações, dentre as quais podem ser mencionadas:

a. A resistência de vários insetos-praga a esses compostos, podendo assim, ocorrer o ressurgimento de determinados insetos em níveis mais altos do que os já existentes; 
b. O aparecimento de pragas secundárias devido ao combate indiscriminado a todo tipo de inseto, ocorrendo dessa forma a exterminação de predadores naturais;

c. E o mais alarmante, é a contaminação ambiental, fazendo com que os pesticidas sejam incorporados a nossa alimentação, causando sérios riscos à saúde (PERES e MOREIRA, 2007).

A solução ideal para o combate aos insetos seria o desenvolvimento de agentes altamente específicos que agissem apenas sobre as espécies nocivas, não permitissem o desenvolvimento de resistência e que não colocassem em risco a preservação do meio ambiente, a saúde do homem e de animais. Frente a essas condições, é importante mencionar que os feromônios ocupam lugar de destaque, por serem substâncias naturais que regulam comportamentos essenciais para a sobrevivência da espécie. É improvável que os insetos desenvolvam algum tipo de resistência a eles, inversamente ao que ocorre com os inseticidas tradicionais. Neste caso, a possibilidade de haver danos ambientais estaria completamente descartada.

\section{A COMUNICAÇÃO ENTRE OS INSETOS E O USO DE FEROMÔNIOS NO CONTROLE DE PRAGAS}

Os insetos, além das formas auditiva, visual e táctil, podem emitir substâncias químicas para se comunicar. A essas substâncias, foi proposto o nome de semioquímico (do grego semeon, "sinal") (VILELA e DELLA LUCIA, 2001). Esses semioquímicos estão relatados na figura 4 e são classificados em duas categorias: Feromônios e Aleloquímicos (ZARBIN e RODRIGUES, 2009).

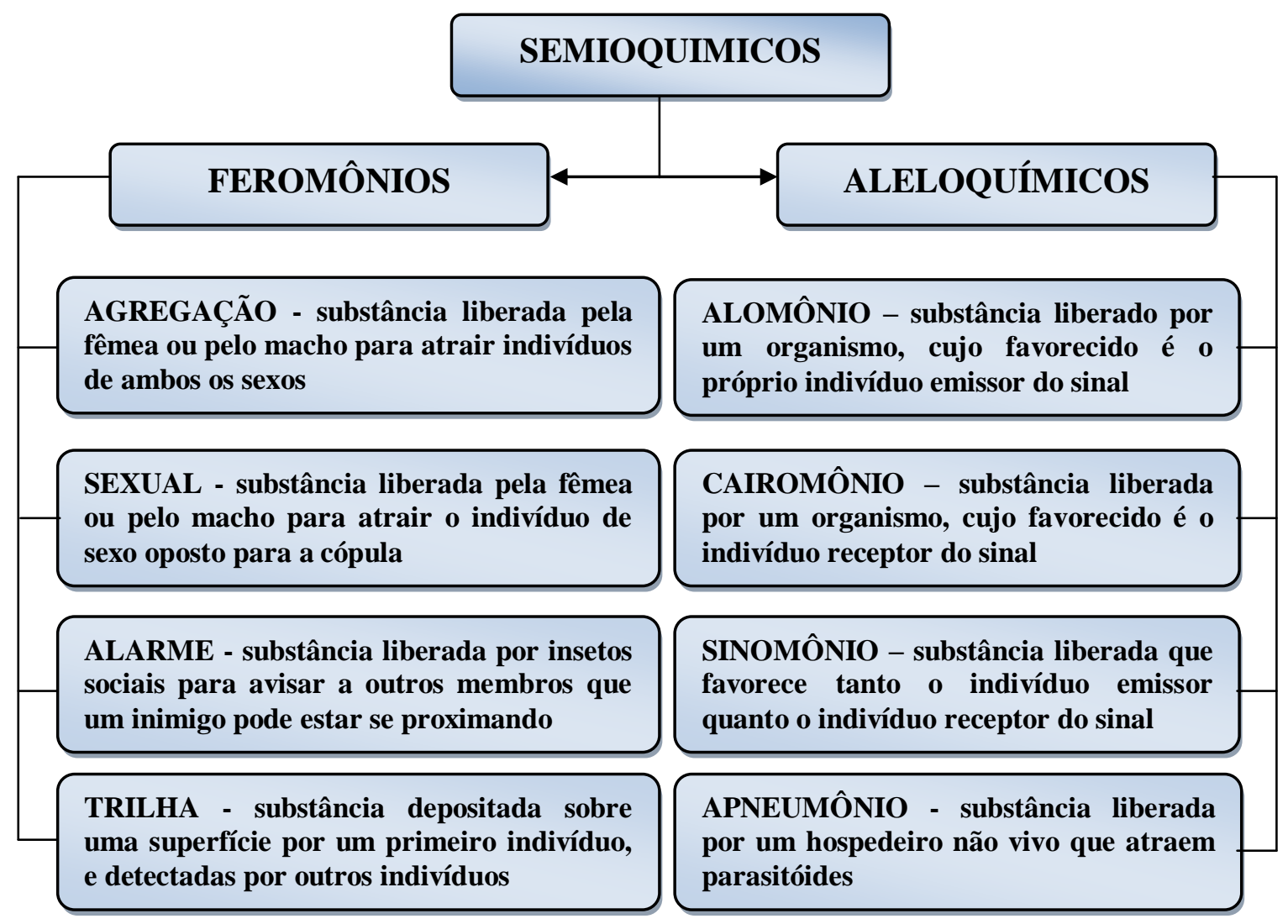

Figura 4 - Classificação dos Semioquímicos. 
Mais modernamente, o uso de feromônios é sempre associado à técnica chamada Manejo Integrado de Pragas (MIP). Neste caso, podem ser utilizados sozinhos ou em conjunto com outros métodos como, o controle biológico, a rotação de culturas e o uso controlado de pesticidas, para detectar, monitorar e controlar os insetos-praga na plantação. Armadilhas contendo feromônios são sensíveis mesmo em baixas densidades populacionais, seletivas, de baixo custo e podem ser facilmente operadas pelos produtores sem nenhum risco eminente.

\section{O GÊNERO METAMASIUS}

Os insetos do gênero Metamasius, ordem Coleoptera, subfamília Curculionidae, possuem ampla distribuição no continente americano, sendo observados desde os Estados Unidos até o Brasil. Estes insetos são encontrados atacando diversas plantas como, Gramineae, Bromeliaceae, Palmaceae, Musaceae, entre outras (ZORZENON et al., 2000).

O Metamasius hemipterus (Linnaeus, 1758) (figura 5) é conhecido como caruncho ou bicudo da cana-de-açúcar. Na América Central, na América do Sul, no Caribe e na África, essa praga está associada a numerosas espécies de plantas como pupunheira, cana-de-açúcar, milho, banana, abacaxi e palmeira (VAURIE, 1966; AMBROGI et al., 2009).

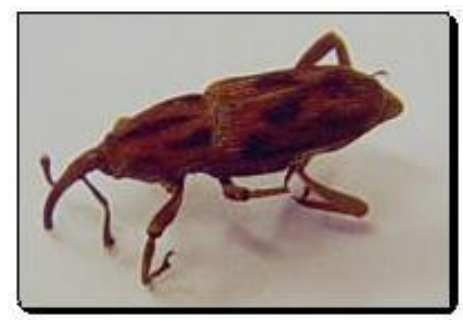

Figura 5 - Adulto do Metamasius hemipterus.

Perez et al. (1997) identificaram os álcoois, 2-Metil-4-octanol (13), 2-Metil-4-heptanol (14), 4-Metil-5-nonanol (15) e 3-Pentanol (16) e as cetonas, 3-Pentanona (17), 2-Metil-4-octanona (18), 2-Metil-4-heptanona (19) e 4-Metil-5-nonanona (20) como possíveis constituintes feromonais. Ramirez-Lucas et al. (1996), além de citarem os álcoois 13, 14 e 15, citam o álcool 5-Nonanol (21) e a hidroxicetona, 3-Hidroxi-4-metil-5-nonanona (22) como possíveis constituintes da mistura feromonal do M. hemipterus L. A figura 6 mostra as estruturas dos principais constituintes do feromônio de agregação deste inseto.

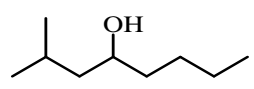

13

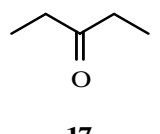

17

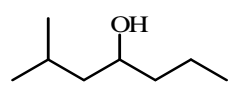

14<smiles>CCCCC(=O)CC(C)C</smiles>

18<smiles>CCCCC(O)CCCC</smiles>

21<smiles>CCCCC(C)C(O)CCC</smiles>

15<smiles>CCCC(=O)CC(C)C</smiles>

19<smiles>CCCCC(=O)C(C)C(O)CC</smiles>

22

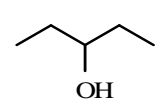

16<smiles>CCCCC(=O)C(C)CCC</smiles>

20

Figura 6 - Estruturas dos principais constituintes do feromônio de agregação de Metamasius hemipterus. 


\section{O GÊNERO RHYNCHOPHORUS}

Desde a década de 1960 tem sido observado larvas do gênero Rhynchophorus, ordem Coleoptera, subfamília Curculionidae, se alimentando de uma grande variedade de plantas das famílias Arecaceae, Gramineae, Caricaceae, Bromeliaceae e Musaceae em quase toda a região tropical do planeta (HAGLEY, 1965), causando assim, sérios prejuízos de importância econômica e/ou ornamental (HILL, 1983). Essas espécies são conhecidas como brocas ou carunchos e os mais comuns em palmeiras são: Rhynchophorus palmarum (Linnaeus, 1764) (figura 7A) e Rhynchophorus cruentatus (Fabricius, 1775) (figura 7B) nas Américas; Rhynchophorus phoenicis (Fabricius, 1801) (figura 7C) na África, Rhynchophorus ferrugineus (Olivier, 1790) (figura 7D) e Rhynchophorus vulneratus (Schoenherr, 1838) (figura 7E), na Ásia, Rhynchophorus. ferrugineus no Oriente Médio e Rhynchophorus bilineatus (Montrouzier, 1857) (figura 7F) nas Ilhas do Pacífico (Oceania) (ROCHAT et al., 1991, 1993; GRIES et al., 1993; HALLETT et al., 1993).

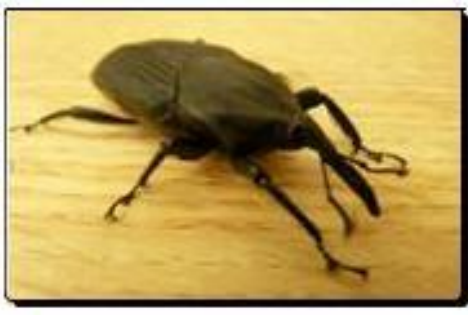

A) R. palmarum. (Foto: Mikael de Lima Freitas)

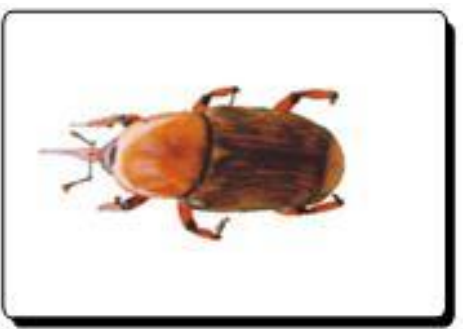

D) R. ferrugineus.

(Fonte:http://naturamediterraneo.com/fo rum/topic.asp? Em 12/04/08).

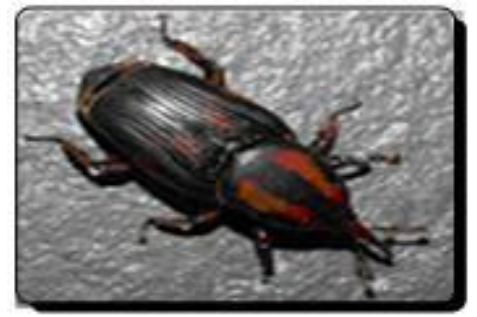

B) $R$. cruentatus.

(Fonte:http://static.fkicr.com/66/16463482 2_df18f7a771.jpg. Em 12/04/08).

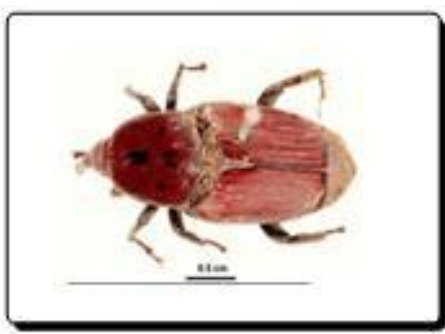

E) R. vulneratus.

(Fonte: http://pikul.lib.ku.ac.th/insect. Em 12/04/08)

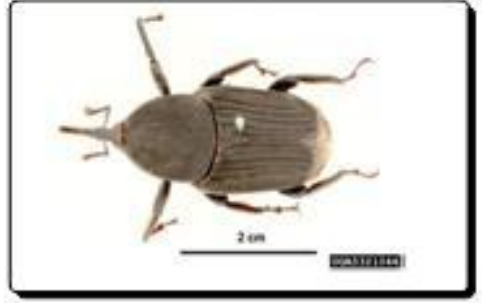

C) R. phoenicis.

(Fonte:

http://padil.gov.au/viewPestDiagno sticImages.aspx?id=952. Em 12/04/08).

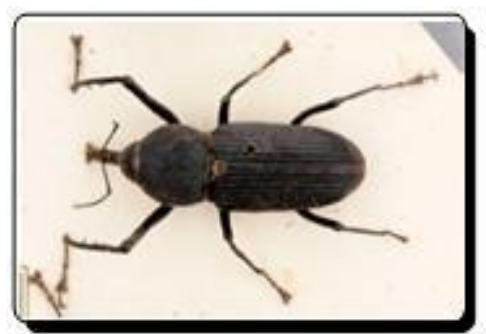

F) $R$. bilineatus.

(Fonte: http://ipmimages.org/images.jpg. Em 12/04/08).

\section{Figura 7 - Adultos do gênero Rhynchophorus}

Estudos de laboratórios e de campo comprovaram que algumas brocas do gênero Rhynchophorus produzem álcoois secundários e ramificados como principais constituintes do feromônio de agregação (PEREZ et al., 1994) como exemplos, podemos descrever o 4-Metil5-nonanol (15) do $R$. ferrugineus, $R$. vulneratus e $R$. bilineatus (HALLETT et al., 1993; ROCHAT et al., 1993), o 6-Metil-2-hepten-4-ol (23) do R. palmarum (ROCHAT et al., 1991), o 3-Metil-4-octanol (24) do R. phoenecis (GRIES et al., 1993; ROCHAT et al., 1993) e o 5Metil-4-octanol (25) do R. cruentatus (PEREZ et al., 1994) (figura 8).<smiles>CCCCC(O)C(C)CCC</smiles>

15<smiles>C/C=C/C(O)CC(C)C</smiles>

23
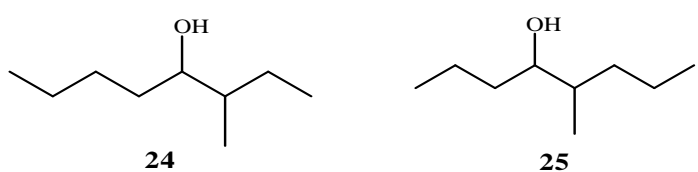

Figura 8 - Principais constituintes do feromônio de agregação de insetos do gênero Rhynchophorus. 
Visto a grande importância dos feromônios destas pragas, este trabalho teve como objetivo padronizar a síntese racêmica em escala preparativa dos álcoois 2-Metil-4-octanol (13), 2Metil-4-heptanol (14), 4-Metil-5-nonanol (15), 5-Nonanol (21), 6-Metil-2-hepten-4-ol (23), 3Metil-4-octanol (24) e 5-Metil-4-octanol (25) e das cetonas 2-Metil-4-octanona (18), 2-Metil4-heptanona (19) e 4-Metil-5-nonanona (20).

\section{PARTE EXPERIMENTAL}

\section{A) PROCEDIMENTO GERAL DE SÍNTESE DOS ÁLCOOIS}

Para a preparação dos alcoóis foi utilizada, com algumas modificações, a metodologia sintética descrita por Grignard (1900) e segue o protocolo geral descrito a seguir:

A uma mistura de magnésio metálico em lascas ativado e THF seco (inicial), foi adicionado um cristal de $\mathrm{I}_{2(\mathrm{~S})}$. Sob agitação e atmosfera de $\mathrm{N}_{2}$, foi adicionada gota-a-gota uma solução do brometo de alquila em THF. Após o término dessa adição, a mistura foi mantida em agitação por 20 minutos entre $5^{\circ} \mathrm{C}$ e $10^{\circ} \mathrm{C}$. Em seguida, foi adicionada gota-a-gota, uma solução do aldeído em THF. Após o término desta adição, a mistura foi mantida em agitação por 18 horas à temperatura ambiente, e posteriormente hidrolisada com $230 \mathrm{~mL}$ de solução de $\mathrm{HC \ell} 10 \% \mathrm{a}$ $0^{\circ} \mathrm{C}$ por cerca de 2 horas. A fase orgânica foi extraída com éter etílico e tratada com água destilada, solução de $\mathrm{NaHCO}_{3} 10 \%$ e solução saturada de $\mathrm{NaC \ell}$. A fase orgânica resultante foi secada com $\mathrm{Na}_{2} \mathrm{SO}_{4}$, filtrada e os solventes evaporados. $\mathrm{O}$ produto bruto obtido foi purificado por destilação sob pressão reduzida. Os dados de RMN ${ }^{1} \mathrm{H}, \mathrm{RMN}{ }^{13} \mathrm{C}$ e CG-EM, confirmaram a pureza e a estrutura de cada álcool.

\section{B) PROCEDIMENTO GERAL DE SÍNTESE DAS CETONAS}

A uma mistura do álcool e ácido acético glacial, adicionou-se gota-a-gota, uma solução de hipoclorito de sódio 2,0-2,5\% ( $\mathrm{NaOC} \ell$ - água sanitária comercial). Em seguida foi adicionada água destilada e a fase orgânica extraída com diclorometano e tratada com solução de $\mathrm{NaHCO}_{3} 10 \%$ e solução saturada de $\mathrm{NaHSO}_{3}$. A mistura foi secada com $\mathrm{Na}_{2} \mathrm{SO}_{4}$, filtrada e os solventes evaporados. O resíduo resultante foi purificado em gel de sílica. Os dados de RMN ${ }^{1} \mathrm{H}, \mathrm{RMN}{ }^{13} \mathrm{C}$ e CG-EM, confirmaram a pureza e a estrutura química de cada cetona.

\section{RESULTADOS E DISCUSSÃO}

\section{A) PREPARAÇÃo dOS ÁlCOOIS}

As observações descritas, para as reações de preparação dos álcoois, foram feitas de modo geral, pois todas as reações apresentaram o mesmo comportamento, variando apenas no que diz respeito aos tempos reacionais, conforme indicados na tabela 1 .

Após a adição do $\mathrm{I}_{2(\mathrm{~s})}$ ao balão, a mistura reacional adquiriu uma coloração marromavermelhada, e à medida que a solução de brometo foi adicionada, observou-se que esta coloração foi gradativamente desaparecendo. Nesta adição, que é exotérmica, foi observada a formação do reagente de Grignard. O tempo de adição variou entre 80 e 100 minutos para as preparações dos alcoóis.

As quantidades de magnésio, THF inicial, brometo de alquila, THF utilizado na primeira adição, aldeído e THF utilizado na segunda adição, assim como os tempos da primeira e segunda adição e o rendimento reacional obtido estão indicados na tabela 1 . 
Tabela 1: Quantidade de reagentes e produtos, rendimentos reacionais e tempo das adições obtidas nas melhores condições experimentais.

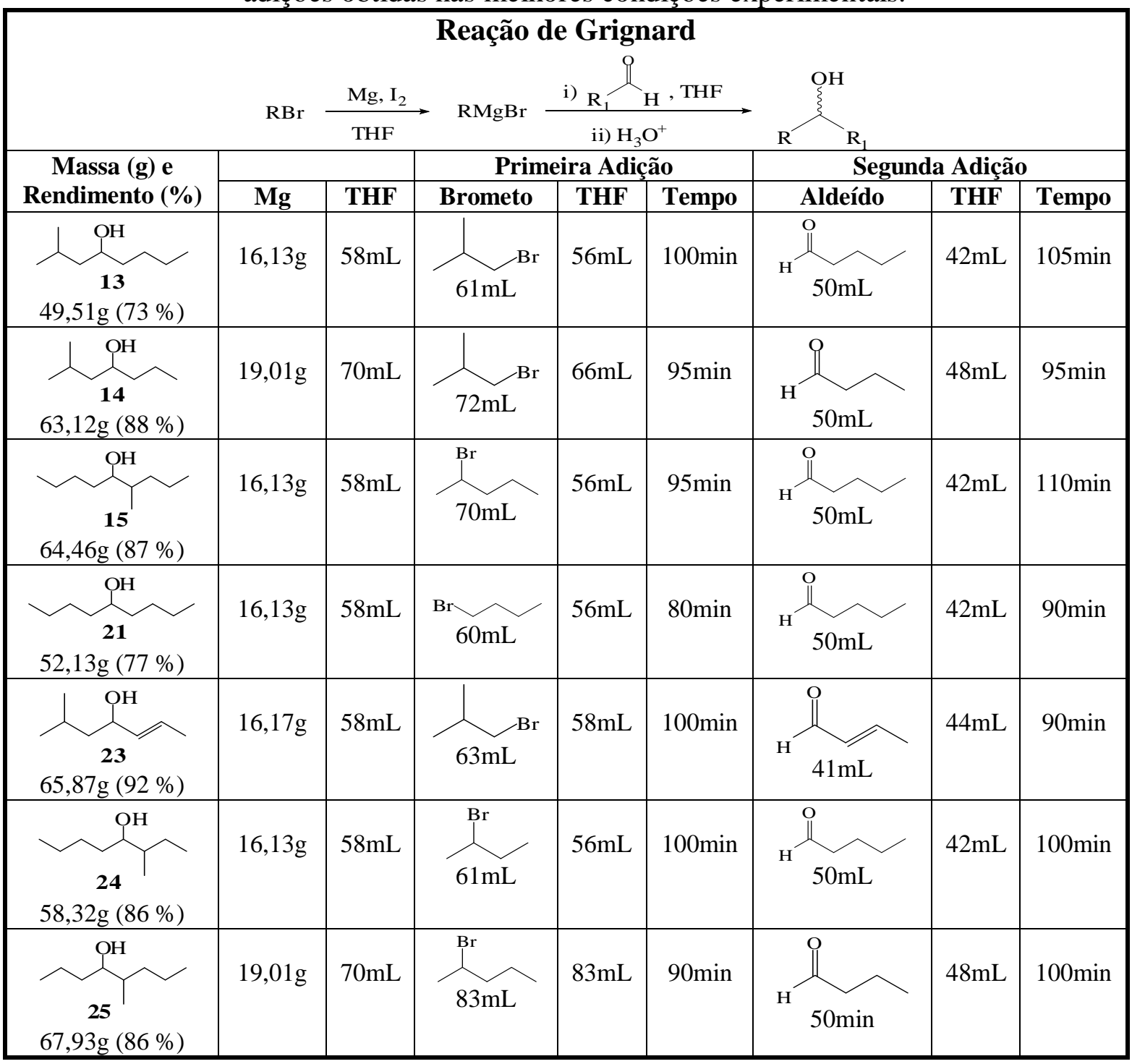

A seguir estão descritos os dados de $\mathrm{RMN}^{1} \mathrm{H}, \mathrm{RMN}{ }^{13} \mathrm{C}$ e CG-EM para os alcoóis sintetizados.

\section{2-Metil-4-octanol (13)}

$\boldsymbol{R M N}{ }^{1} \boldsymbol{H}\left(\mathbf{C D C l}_{3}, \boldsymbol{\delta}\right.$, TMS, $\left.400 \mathrm{MHz}\right): 0,90(3 \mathrm{H}, \mathrm{d}, J=5,0 \mathrm{~Hz}) ; 0,91(3 \mathrm{H}, \mathrm{t}, J=7,0 \mathrm{~Hz})$; $0,92(3 \mathrm{H}, \mathrm{d}, J=5,0 \mathrm{~Hz}) ; 1,19-1,44(8 \mathrm{H}, \mathrm{m}) ; 1,73-1,81(1 \mathrm{H}, \mathrm{m}) ; 3,58-3,67(1 \mathrm{H}, \mathrm{m})$.

$\boldsymbol{R M N}{ }^{13} \boldsymbol{C}\left(\mathrm{CDCl}_{3}, \boldsymbol{\delta}, \mathbf{T M S}, \mathbf{1 0 0} \mathrm{MHz}\right): 13,87 ; 21,87 ; 22,63 ; 23,29 ; 24,35 ; 27,70 ; 37,61$; 46,$62 ; 69,50$.

CG-EM (70 eV) $\boldsymbol{m} / z: 142\left(\mathrm{M}^{+}-2\right)(0,13) ; 111(1,25) ; 98(0,70) ; 87(46,61) ; 69(100,00) ; 57$ $(19,32) ; 45(41,93) ; 43(69,80) ; 41(85,04)$.

\section{2-Metil-4-heptanol (14)}

$\boldsymbol{R M N}{ }^{1} \boldsymbol{H}\left(\mathbf{C D C l}_{3}, \boldsymbol{\delta}, \mathbf{T M S}, 400 \mathrm{MHz}\right): 0,89-0,94$ (9H, m); 1, 18-1,48 (6H, m); 1,73-1,83 (1H, m); 2,29 (1H, sl); 3,58-3,69 (1H, m). 
$\boldsymbol{R M N}{ }^{13} \boldsymbol{C}\left(\mathrm{CDCl}_{3}, \boldsymbol{\delta}\right.$, TMS, $\left.100 \mathrm{MHz}\right): 14,18 ; 18,92 ; 22,16 ; 23,54 ; 24,61 ; 40,39 ; 46,93$; 69,40 .

$\boldsymbol{C G}-\boldsymbol{E M}(70 \mathrm{eV}) \boldsymbol{m} / \boldsymbol{z}: 128\left(\mathrm{M}^{+}-2\right)(0,31) ; 112(1,86) ; 97(0,78) ; 87(28,48) ; 73$ (53,16); 69 $(86,09) ; 55(100,00) ; 43(81,83)$.

\section{4-Metil-5-nonanol (15)}

$\boldsymbol{R M N}{ }^{1} \boldsymbol{H}\left(\mathrm{CDCl}_{3}, \boldsymbol{\delta}, \mathbf{T M S}, 400 \mathrm{MHz}\right): 0,89-0,97$ (9H, m); 1,12-1,59 (9H, m); 2,61 (1H, sl); 3,42-3,47 (1H, m); 3,48-3,52 (1H, m).

$\boldsymbol{R M N}{ }^{13} \boldsymbol{C}\left(\mathrm{CDCl}_{3}, \boldsymbol{\delta}, \mathbf{T M S}, \mathbf{1 0 0} \mathbf{M H z}\right): 13,61 ; 14,02 ; 14,07 ; 14,32 ; 14,36 ; 15,17 ; 20,50$; 20,$52 ; 22,88 ; 22,89 ; 28,47 ; 28,59 ; 33,01 ; 34,18 ; 34,30 ; 35,65 ; 38,01 ; 38,66 ; 75,05 ; 75,86$.

CG-EM (70 eV) $\boldsymbol{m} / \boldsymbol{z}: 157\left(\mathrm{M}^{+}-1\right)(0,11) ; 140(1,13) ; 127(0,11) ; 114(0,53) ; 101$ (12,84); 87 $(43,49) ; 69(100,00) ; 55(39,64) ; 43(65,46) ; 41(92,08)$.

\section{5-Nonanol (21)}

$\boldsymbol{R M N}{ }^{1} \boldsymbol{H}\left(\mathbf{C D C l}_{3}, \boldsymbol{\delta}\right.$, TMS, $\left.400 \mathrm{MHz}\right): 0,91(6 \mathrm{H}, \mathrm{t}, J=7,0 \mathrm{~Hz}) ; 1,29-1,47(12 \mathrm{H}, \mathrm{m}) ; 2,01$ $(1 \mathrm{H}, \mathrm{sl}) ; 3,54-3,60(1 \mathrm{H}, \mathrm{m})$.

$\boldsymbol{R M N}{ }^{13} \boldsymbol{C}\left(\mathrm{CDCl}_{3}, \boldsymbol{\delta}, \mathbf{T M S}, \mathbf{1 0 0} \mathrm{MHz}\right): 14,13 ; 22,86 ; 27,95 ; 37,24 ; 71,96$.

CG-EM (70 eV) $\boldsymbol{m} / \boldsymbol{z}: 143\left(\mathrm{M}^{+}-1\right)(0,24) ; 126(1,61) ; 114(0,01) ; 97(0,60) ; 87(48,37) ; 69$ $(100,00) ; 57(20,34) ; 41(84,45)$.

\section{6-Metil-2-hepten-4-ol (23)}

$\boldsymbol{R M N}{ }^{1} \boldsymbol{H}\left(\mathrm{CDCl}_{3}, \boldsymbol{\delta}, \mathbf{T M S}, 400 \mathrm{MHz}\right): 0,90(3 \mathrm{H}, \mathrm{d}, J=6,0 \mathrm{~Hz}) ; 0,92(3 \mathrm{H}, \mathrm{d}, J=6,0 \mathrm{~Hz})$; $1,31(2 \mathrm{H}, \mathrm{m}) ; 1,49(1 \mathrm{H}, \mathrm{sl}) ; 1,60(1 \mathrm{H}, \mathrm{m}) ; 1,71\left(3 \mathrm{H}, \mathrm{dd}, J_{1}=0,6 \mathrm{~Hz}, J_{2}=1,0 \mathrm{~Hz}\right) ; 4,11(1 \mathrm{H}, \mathrm{q}$, $J=7,0 \mathrm{~Hz}) ; 5,48\left(1 \mathrm{H}, \mathrm{qd}, J_{1}=1,0 \mathrm{~Hz}, J_{2}=7,0 \mathrm{~Hz}\right) ; 5,65\left(1 \mathrm{H}, \mathrm{qd}, J_{1}=1,0 \mathrm{~Hz}, J_{2}=7,0 \mathrm{~Hz}\right)$.

$\boldsymbol{R M N}{ }^{13} \boldsymbol{C}\left(\mathrm{CDCl}_{3}, \boldsymbol{\delta}, \mathbf{T M S}, \mathbf{1 0 0} \mathrm{MHz}\right): 22,37 ; 22,43 ; 23,60 ; 28,37 ; 41,62 ; 69,01 ; 129,73$; 135,53 .

$\boldsymbol{C G}-\boldsymbol{E M}(70 \mathrm{eV}) \boldsymbol{m} / \boldsymbol{z}: 128\left(\mathrm{M}^{+}\right)(0,55) ; 95(16,53) ; 86(8,88) ; 71(100,00) ; 57(14,95) ; 43$ $(51,56)$.

\section{3-Metil-4-octanol (24)}

$\boldsymbol{R M N}{ }^{1} \boldsymbol{H}\left(\mathrm{CDCl}_{3}, \boldsymbol{\delta}, \mathbf{T M S}, 400 \mathrm{MHz}\right): 0,86-0,93$ (9H, m); 1,20-1,57 (9H, m); 2,63 (1H, sl); 3,40-3,45 (1H, m); 3,48-3,52 (1H, m).

$\boldsymbol{R M N}{ }^{13} \boldsymbol{C}\left(\mathrm{CDCl}_{3}, \boldsymbol{\delta}, \mathbf{T M S}, \mathbf{1 0 0} \mathrm{MHz}\right): 11,85 ; 12,02 ; 13,31 ; 14,16 ; 14,22 ; 14,87 ; 22,95$; 22,$97 ; 24,75 ; 26,15 ; 28,44 ; 28,62 ; 33,20 ; 34,32 ; 40,11 ; 40,68 ; 75,01 ; 75,88$.

$\boldsymbol{C G}-\boldsymbol{E M}(70 \mathrm{eV}) \boldsymbol{m} / \boldsymbol{z}: 142\left(\mathrm{M}^{+}-2\right)(0,43) ; 126(1,68) ; 114(0,05) ; 97(2,04) ; 87(49,48) ; 69$ $(99,08) ; 57(31,47) ; 41(100,00)$. 


\section{5-Metil-4-octanol (25)}

$\boldsymbol{R M N}{ }^{1} \boldsymbol{H}\left(\mathbf{C D C l}_{3}, \boldsymbol{\delta}\right.$, TMS, $\left.400 \mathrm{MHz}\right): 0,86(3 \mathrm{H}, \mathrm{d}, J=7,0 \mathrm{~Hz}) ; 0,88(3 \mathrm{H}, \mathrm{d}, J=7,0 \mathrm{~Hz})$; $0,90(3 \mathrm{H}, \mathrm{t}, J=7,0 \mathrm{~Hz}) ; 0,93(3 \mathrm{H}, \mathrm{t}, J=7,0 \mathrm{~Hz}) ; 1,06-1,56(18 \mathrm{H}, \mathrm{m}) ; 1,76(2 \mathrm{H}, \mathrm{sl}) ; 3,43-3,47$ $(1 \mathrm{H}, \mathrm{m}) ; 3,49-3,53(1 \mathrm{H}, \mathrm{m})$.

$\boldsymbol{R M N}{ }^{13} \boldsymbol{C}\left(\mathbf{C D C l}_{3}, \boldsymbol{\delta}, \mathbf{T M S}, \mathbf{1 0 0} \mathbf{M H z}\right): 13,60 ; 14,19 ; 14,21 ; 14,37 ; 14,42 ; 15,22,19,35 ;$ 19,$49 ; 20,47 ; 20,51 ; 34,21,35,54 ; 35,62,36,68 ; 37,99,38,63 ; 74,95,75,80$.

CG-EM (70 eV ) $\boldsymbol{m} / \boldsymbol{z}: 143\left(\mathrm{M}^{+}-1\right)(0,07) ; 126(1,22) ; 111(0,05) ; 101$ (7,97); 83 (12,57); 73 $(41,96) ; 55(100,00) ; 43(60,64)$.

\section{B) PREPARAÇÃO DAS CETONAS}

As reações de oxidação dos álcoois, 2-Metil-4-octanol (13), 2-Metil-4-heptanol (14) e 4Metil-5-nonanol (15) com NaOCl, para obtenção das cetonas 2-Metil-4-octanona (18), 2Metil-4-heptanona (19) e 4-Metil-5-nonanona (20), foi realizada sem problemas e constituiu uma boa alternativa para a síntese em escala preparativa. A tabela 2 indica as quantidades de reagentes e produtos, bem como o rendimento para cada reação.

Tabela 2: Quantidades de reagentes, produtos e rendimento reacional.

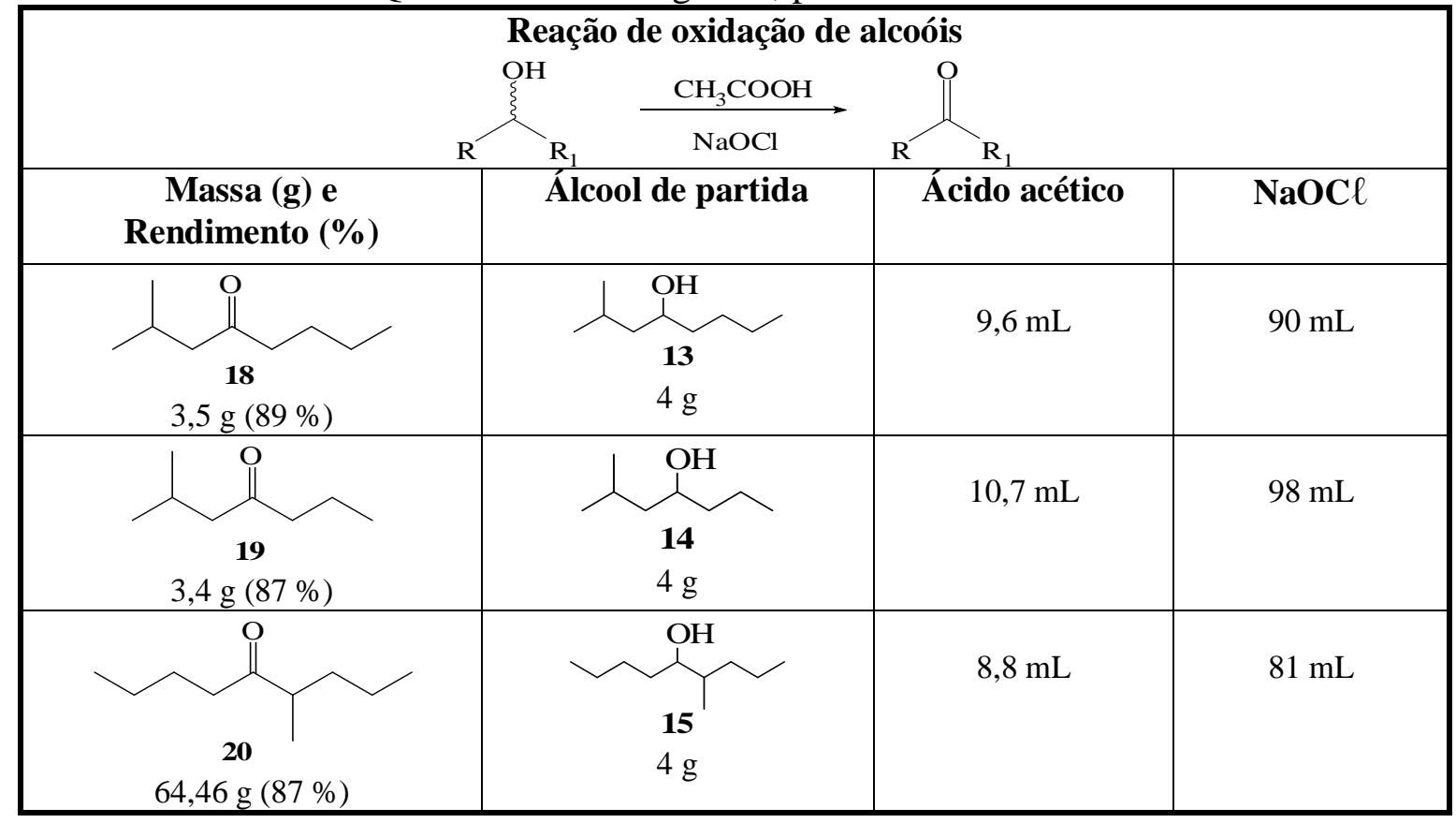

A seguir estão os dados de $\mathrm{RMN}{ }^{1} \mathrm{H}, \mathrm{RMN}^{13} \mathrm{C}$ e CG-EM obtidos para cada cetona sintetizada.

\section{2-Metil-4-octanona (18)}

$\boldsymbol{R M N}{ }^{1} \boldsymbol{H}\left(\mathbf{C D C l}_{3}, \boldsymbol{\delta}\right.$, TMS, $\left.400 \mathrm{MHz}\right): 0,82(3 \mathrm{H}, \mathrm{t}, J=6,5 \mathrm{~Hz}) ; 0,83(6 \mathrm{H}, \mathrm{d}, J=6,5 \mathrm{~Hz})$; 1,18-1,27 (2H, m); 1,43-1,50 (2H, m); 2,02-2,09 (1H, m); 2,19 (2H, d, $J=7,0 \mathrm{~Hz})$.

$\boldsymbol{R M N}{ }^{13} \boldsymbol{C}\left(\mathrm{CDCl}_{3}, \boldsymbol{\delta}, \mathbf{T M S}, \mathbf{1 0 0} \mathrm{MHz}\right): 13,66 ; 22,18 ; 22,38 ; 24,39 ; 25,70 ; 42,84 ; 51,61$; 210,85 .

CG-EM (70 eV) m/z: $142\left(\mathrm{M}^{+}\right)$(7,69); $127(2,67) ; 113(1,04) ; 100(4,92) ; 85(62,89) ; 71$ $(2,30) ; 58(46,35) ; 57(100,00) ; 41(40,31)$. 
2-Metil-4-heptanona (19)

$\boldsymbol{R M N}{ }^{1} \boldsymbol{H}\left(\mathbf{C D C l}_{3}, \boldsymbol{\delta}, \mathbf{T M S}, 400 \mathrm{MHz}\right): 0,77-0,79(9 \mathrm{H}, \mathrm{m}) ; 1,43-1,49$ (6H, m); 1,97-2,04 (1H, m); 2,14 (2H, d, $J=7,0 \mathrm{~Hz}) ; 2,23(2 \mathrm{H}, \mathrm{t}, J=7,5 \mathrm{~Hz})$.

$\boldsymbol{R M N}{ }^{13} \boldsymbol{C}\left(\mathrm{CDCl}_{3}, \boldsymbol{\delta}, \mathbf{T M S}, \mathbf{1 0 0} \mathrm{MHz}\right): 13,58 ; 17,05 ; 22,42 ; 24,43 ; 45,07 ; 51,68 ; 210,77$.

CG-EM (70 eV) m/z: $128\left(\mathrm{M}^{+}\right)(14,67) ; 113(4,52) ; 100(0,02) ; 88(0,02) ; 71$ (54,91); 58 $(29,25) ; 57(100,00) ; 43(91,94)$.

\section{4-Metil-5-nonanona (20)}

$\boldsymbol{R M N}{ }^{1} \boldsymbol{H}\left(\mathrm{CDCl}_{3}, \boldsymbol{\delta}\right.$, TMS, $\left.400 \mathrm{MHz}\right): 0,72-0,80(6 \mathrm{H}, \mathrm{m}) ; 0,91(3 \mathrm{H}, \mathrm{d}, J=7,0 \mathrm{~Hz}) ; 1,10-$ $1,21(4 \mathrm{H}, \mathrm{m}) ; 1,37-1,50(4 \mathrm{H}, \mathrm{m}) ; 2,29(2 \mathrm{H}, \mathrm{t}, J=7,5 \mathrm{~Hz}) ; 2,30(2 \mathrm{H}, \mathrm{t}, J=7,5 \mathrm{~Hz}) ; 2,37-2,44$ $(1 \mathrm{H}, \mathrm{m})$.

$\boldsymbol{R M N}{ }^{13} \boldsymbol{C}\left(\mathrm{CDCl}_{3}, \boldsymbol{\delta}, \mathbf{T M S}, \mathbf{1 0 0} \mathrm{MHz}\right): 13,60 ; 13,81 ; 16,04 ; 20,24 ; 22,16 ; 25,56 ; 34,17$; 40,$60 ; 45,80 ; 214,54$.

CG-EM (70 eV) $\boldsymbol{m} / \boldsymbol{z}: 156\left(\mathrm{M}^{+}\right)(1,22) ; 141(0,10) ; 127(1,78) ; 114(24,36) ; 99(8,07) ; 85$ $(75,74) ; 71(62,14) ; 57(100,00) ; 43(74,36)$.

\section{CONCLUSÕES}

A síntese em escala preparativa dos álcoois ( \pm )-2-Metil-4-octanol (13), ( \pm )-2-Metil-4heptanol (14), ( \pm )-4-Metil-5-nonanol (15), $( \pm)-5-$ Nonanol (21), ( \pm )-3-Metil-4-octanol (24),

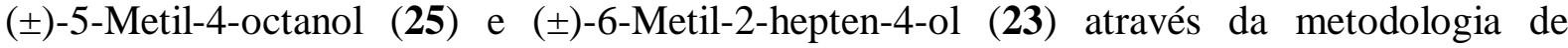
Grignard foi realizada com sucesso, sendo satisfatória para a obtenção dos referidos compostos com elevados rendimentos reacionais.

A oxidação dos álcoois ( \pm )-2-Metil-4-octanol (13), ( \pm )-2-Metil-4-heptanol (14), ( \pm )-4-Metil5-nonanol (15) com solução de hipoclorito de sódio (água sanitária comercial) para obtenção das cetonas 2-Metil-4-octanona (18), 2-Metil-4-heptanona (19) e ( \pm )-4-Metil-5-nonanona (20) constitui uma alternativa técnica e economicamente viável.

Cabe salientar, que o referido trabalho visou o desenvolvimento científico e tecnológico regional na área de Química, em particular na Síntese Orgânica, pois além de fornecer os produtos químicos desejados, qualifica e re-qualifica os estudantes e pesquisadores envolvidos, no que diz respeito à formação de recursos humanos no desenvolvimento de Química fina aplicada.

\section{REFERÊNCIAS}

1. AMBROGI, B. G.; VIDAL, D. M. e ZARBIN, P. H .G. Feromônios de agregação em Curculionidae (Insecta: Coleoptera) e sua implicação taxonômica. Química Nova, v.32, n.8, p.2151-2158, 2009.

2. DAMON, A. A review of the biology and control of the coffee berry borer, Hypothenemus hampei (Coleoptera: Scolytidae), Bulletin of Entomological Research, v.90, p.453-465, 2000. 
3. DAY, K. R. Cooperative Extension Work in Agriculture and Home Economics, U.S. Department of Agriculture, University of California, Tulare County Cooperating, p.1-4, Oct., 2000.

4. GRIES, G.; GRIES, R.; PEREZ, A. L.; OEHLSCHLAGER, A. C.; GONZALES, L. M.; PIERCE, H. D.; KOUDA, M.; ZEBEYOU, M. e NANOU, N. Aggregation pheromone of the African palm weevil, Rhynchophorus phoenicis F. Naturwissenschaften, v.80, p.90-91, 1993.

5. GRIGNARD, V. Comptes Rendus, v.130, p.1322, 1900.

6. HAGLEY, E. A. C. On the life history and habitats of the palm weevil Rhynchophorus palmarum (L), Annals of the Entomological Society of America, v.58, n.1, p.22-28, 1965.

7. HALLETT, R. H.; GRIES, R.; BORDEN, J. H.; CZYZEWSKA, E.; OEHLSCHLAGER, A. C.; PIERCE, H. D.; Jr., ANGERILLI, N. D. P. e RAUF, A. Aggregation pheromones of two Asian palm weevils, Rhynchophorus ferrugineus and Rhynchophorus vulneratus. Naturwissenschaften, v.80, p.328-331, 1993.

8. HILL, D. S. Agricultural Insect Pests of the Tropics and Their Control. Cambridge University Press, New York, 1983.

9. <http://ipmimages.org/images.jpg>. Em 12/04/08.

10. <http://naturamediterraneo.com/forum/topic.asp?> Em 12/04/08.

11. <http://padil.gov.au/viewPestDiagno sticImages.aspx?id=952>. Em 12/04/08.

12. <http://pikul.lib.ku.ac.th/insect>. Em 12/04/08.

13. <http://static.flickr.com/66/164634822_df18f7a771.jpg>. Em 12/04/08.

14. MARICONI, F. A. M. Inseticidas e seu emprego no controle às pragas, Editora Nobel, $7^{a}$ edição, São Paulo, p.95, 1988.

15. PERES, F. e MOREIRA, J. C. Saúde e ambiente em sua relação com o consumo de agrotóxicos em um pólo agrícola do Estado do Rio de Janeiro, Brasil. Cad. Saúde Pública, Rio de Janeiro, 23 Sup 4:S612-S621, 2007.

16. PEREZ, A. L.; GRIES, G.; GRIES, R.; GIBLIN-DAVIS, R. M. e OEHLSCHLAGER, A. C. Pheromone chirality of African palm weevil, Rhynchophorus phoenicis (F.) and palmetto weevil, Rhynchophorus cruentatus (F.) (Coleoptera: Curculionidae), Journal of Chemical Ecology, v.20, n.10, p.2653-2671, 1994.

17. PEREZ, A. L.; CAMPOS, Y.; CHINCHILlA, C. M.; OEHLSCHLAGER, A. C.; GRIES, G.; GRIES, R.; GIBLIN-DAVIS, R. M.; CASTRILLO, G.; PEÑA, J. E.; DUNCAN, R. E.; GONZALEZ, L. M.; PIERCE, H. D.; McDONALD, R. e ANDRADE, R. Aggregation Pheromones and Host Kairomones of West Indian Sugarcane Weevil, Metamasius hemipterus, Journal of Chemical Ecology, v.23, n.4, p.869-887, 1997.

18. RAMIREZ-LUCAS, P.; MALOSSE, C.; DUEROT, P. H.; LETTERE, M. e ZAGATTI, $P$. Chemical Identification, Electrophysiological and Behavioral Activities of the Pheromone of Metamasius hemipterus (Coleoptera: Curculionidae), Bioorganic \& Medicinal Chemistry, v.4, n.3, p.323-330, 1996.

19. ROCHAT, D.; MAlOSSE, C.; LETTERE, M.; DUEROT, P. H.; ZAGATTI, P.; RENOU, M. e DESCOINS, C. Male produced aggregation pheromone in American palm weevil, Rhynchophorus palmarum (L.) (Coleoptera: Curculionidae). Journal of Chemical Ecology, v.17, n.11, p.2117-2141, 1991. 
20. ROCHAT, D.; DESCOINS, C.; MALOSSE, C.; NAGNAN, P., ZAGATTI, P.; AKAMOU, F. e MARIAU, D. Ecologie chimique des charançons des palmiers, Rhynchophorus ssp (Coleoptera). Oleagineux, v.48, p.225-236, 1993.

21. SANTOS, V. M. R.; DONNICI, C. L.; DACOSTA, J. B. N. e CAIXEIRO, J. M. R. 2007. Compostos organofosforados pentavalentes: histórico, métodos sintéticos de preparação e aplicações como inseticidas e agentes antitumorais. Química Nova, v.30, n.1, p.159-170, 2007.

22. TEIXEIRA, C. D.; SOUZA, O. e COSTA, J. N. M. Frutos de Café "Conilon" Brocados por Hypothenemus hampei (Ferrari) (Coleoptera: Scolytidae): Qual a Importância de sua Queda no Decorrer da Fase de Frutificação? Neotropical Entomology, v.35, n.3, p.390-394, 2006.

23. VAURIE, P. A revision of the Neotropical genus Metamasius (Coleoptera, Curculionidae, Rhynchophorinae). Species groups I and II. Bulletin of the American Museum of Natural History, v.131, p.213-337, 1966.

24. VEIGA, M. M.; SILVA, D. M.; VEIGA, L. B. E. e FARIA, M. V. C. Análise da contaminação dos sistemas hídricos por agrotóxicos numa pequena comunidade rural do Sudeste do Brasil. Caderno de Saúde Publica, Rio de Janeiro, v.22, n.11, p.2391-2399, 2006.

25. VILELA, E. F. e DELLA LUCIA, T. M. C. Introdução aos Semioquímicos e Terminologia. In: VILELA, E. F. e DELLA LUCIA, T. M. C. eds. Feromônios de Insetos: Biologia, Química e Emprego no Manejo de Pragas, ${ }^{a}$ edição, Ribeirão Preto: Holos, p.9-12, 2001.

26. WARE, G. The Pesticide Book, Thomson Publications: Fresno, CA, p.340, 1989.

27. ZARBIN. P. H. e ROGRIGUES, M. A. C. M. Feromônios de insetos: tecnologia e desafios para uma agricultura competitiva no Brasil. Química Nova, v.32, n.3, p. 722-731, 2009.

28. ZORZENON, F. J.; BERGMANN, E. C. e BICUDO, J. E. Primeira ocorrência de Metamasius hemipterus (Linnaeus, 1958) e Metamasius ensirostris (Germar, 1928) (Coleoptera: Curculionidae) em palmiteiro dos gêneros Euterpe e Bactris (Arecaceae) no Brasil. Arquivo do Instituto Biológico, São Paulo, v.67, n.2, p.265-268, 2000 . 